\title{
Does smoking cessation reduce the risk of restenosis following coronary angioplasty?
}

Before the widespread use of coronary stents, restenosis occurred in around one third of lesions dilated by percutaneous transluminal coronary angioplasty (PTCA). ${ }^{1}$ The actual rates reported in individual case series vary widely depending on patient selection, the definition of restenosis applied, duration of follow up, completeness of angiographic follow up, and whether or not quantitative methods of assessment are used.

Although infrequently resulting in myocardial infarction or death, ${ }^{12}$ restenosis is nonetheless an important occurrence since it is associated with the recurrence or deterioration of symptoms and, in some patients, necessitates further attempts at revascularisation.

PTCA does not prevent continued progression of the underlying atherosclerotic disease which may result in further stenoses both at the dilatation site and elsewhere in the coronary arterial system. Furthermore, use of balloon dilatation has been implicated as increasing the risk of early restenosis through both direct and indirect effects. Direct intimal injury at or near the site of dilatation induces fibrocellular proliferation which encourages the deposition of platelets and accelerates formation of atherosclerotic lesions. ${ }^{3}$ Balloon dilatation also produces atherogenic effects through the liberation of activated cells and the release of growth promoting factors. ${ }^{5}$

Cigarette smoking is associated with the development and progression of coronary arterial disease. In their retrospective cohort study Violaris et al demonstrate that, on average, current smokers undergo coronary angioplasty three years earlier than ex-smokers, who in turn undergo it three years earlier than non-smokers. ${ }^{6}$ Smokers also possess a different risk profile at the time of angioplasty, being more likely to have peripheral arterial disease or a history of myocardial infarction, but less likely to be diabetic or hypertensive. ${ }^{6}$

One might expect cigarette smoking to affect the risk of disease progression and restenosis following angioplasty because of its known effects on endothelial and platelet function. As a result, patients are routinely advised to cease smoking before coronary angioplasty. However, published studies on the association between smoking and outcome following coronary angioplasty have reported conflicting results.

Violaris et al report a retrospective analysis of 2948 patients recruited to either the active or placebo arms of four randomised trials with negative results. ${ }^{6}$ They demonstrate no significant difference between current, ex- and non-smokers in the rate of restenosis at six months follow up on both univariate and multivariate analysis. The combined end point of repeat revascularisation, myocardial infarction, and death is also not significantly associated with smoking status. This study corroborates the negative results published previously by a number of other investigators. Macdonald et $a l^{7}$ and Gurlek et al reported no significant association between smoking at the time of coronary angioplasty and subsequent restenosis. Similarly Benchimol et al reported no association between cumulative tobacco consumption up to the time of follow up and restenosis following initially successful coronary angioplasty. ${ }^{9}$

By contrast, a number of other published studies have reported beneficial effects from smoking cessation. Myler et al reported a significant association between current smoking and restenosis at six months follow up in a study of 494 consecutive patients who underwent multi-lesion dilatations. ${ }^{10}$ In a retrospective study of 160 patients who achieved primary success following coronary angioplasty, Galan et al reported significantly higher restenosis rates in those who continued to smoke compared to those who stopped following the procedure. ${ }^{11}$ The association between continued smoking and restenosis remained after adjustment for other covariates on multivariate analysis. In a prospective cohort study of 368 men, Jorgensen et al demonstrated improvements in exercise capacity two and 19 weeks following coronary angioplasty in both smokers and non-smokers. ${ }^{12}$ However, the improvements observed were significantly greater among non-smokers.

The lack of consensus is further compounded by the beneficial effects of continued smoking reported in a minority of studies. In a multivariate analysis of 742 successfully dilated lesions, Melkerk et al showed that a history of cigarette smoking was associated with an independent protective effect on absolute luminal loss at six months follow up. ${ }^{13}$ Similarly, Arora et al reported nonsignificantly lower restenosis rates in those who smoked at the time of coronary angioplasty compared with those who had ceased, and those who smoked at the time of follow-up compared with those who had ceased. ${ }^{14}$

The lack of consensus in the published literature can be attributed largely to a number of methodological problems and inconsistencies. Analyses of the association between smoking and restenosis have often taken the form of substudies within studies designed primarily to examine other hypotheses. They are usually only of sufficient power to attempt to disprove the null hypothesis rather than prove it. Therefore, the published negative results may merely reflect type I statistical errors. In the study by Violaris et al, a difference in restenosis rate of less than $20 \%$ would not have been detected as significant. ${ }^{6}$ Those studies undertaken retrospectively may be subject to recall and recording bias.

As the study by Violaris et al showed, smoking status is associated with other case mix factors. ${ }^{6}$ Despite this, a number of studies have failed to adjust for potential confounding factors in analysing the association between smoking status and restenosis. The unit of analysis varies 
between studies, with some using patients and others individual lesions. In the latter individual patients may contribute more than one lesion to the study, thereby increasing the risk of failing to adjust for patient specific confounders.

Most studies treat smoking status as a categorical variable. However, a minority assess cumulative tobacco consumption using pack years. In the former type of studies, the categorisation of ex-smokers is inconsistent. In some studies they are recognised as a distinct category. However, in others they may be included with either current or non-smokers. Smoking status is sometimes assessed at the time of angioplasty and sometimes at the time of follow up. Therefore, those who smoke at the time of angioplasty but subsequently cease may be classified as either current or ex-smokers. Objective confirmation of smoking status is uncommon and, in light of the stigma associated with the activity, reliance on patient histories may result in reporting bias.

Definitions of restenosis vary between studies making comparison of results difficult. Some studies use a binary end point based on either the absolute luminal diameter or the percentage change in diameter. In others the luminal diameter is treated as a continuous variable. Angiographic follow up is generally only undertaken on a subgroup of patients who report symptomatic deterioration, thereby introducing possible selection bias. Also, quantitative assessment of lesions is not used universally.

Advising patients who require coronary angioplasty to stop smoking indefinitely seems intuitively sensible, and will produce additional benefits to any direct effect on the coronary arteries. However, published evidence to support a specific association between continued smoking and restenosis is generally lacking. Further research is required to confirm or refute such an association and inconsistencies in definitions and methodologies need to be addressed.
Department of Medical Cardiology

JILL P PELL

University of Glasgow

Glasgow Royal Infirmary

10 Alexandra Parade

Glasgow G31 2ER, UK

email: jill.pell@glasgow-hb.scot.nhs.uk

1 Meier B. Restenosis after coronary angioplasty: review of the literature Eur Heart $\mathcal{F}$ 1988;9:1-6.

2 Kent KM, Bentivoglio LG, Block PC, et al. Long-term efficacy of percutaneous transluminal coronary angioplasty (PTCA): report from the National Heart, Lung, and Blood Institute PTCA registry. Am $\mathcal{F}$ Cardiol 1984;53:27C-31C

3 Waller BF, Pinkerton A, Foster LN. Morphologic evidence of accelerated left main coronary artery stenosis: a late complication of percutaneous transluminal balloon angioplasty of the proximal left anterior descending coronary artery. $₹ \mathrm{Am}$ Coll Cardiol 1987;9:1019-23.

4 Harker LA. Role of platelets and thrombosis in mechanisms of acute occlusion and restenosis after angioplasty. Am f Cardiol 1987;60:20B-28B.

5 Chesebro JH, Lam JYT, Badimon L, et al. Restenosis after arterial angioplasty: a hemorheologic response to injury. Am $f$ Cardiol 1987;60: angioplast

6 Violaris AG, Melkert R, Ozaki Y, et al. Influence of past and present smoking habits on short term (six month) clinical and angiographic outcome after successful coronary angioplasty. Heart 2000;84:299-306.

7 Macdonald RG, Henderson MA, Hirshfeld JW, et al. Patient-related variables and restenosis after percutaneous transluminal coronary angioplasty-a report from the M-HEART group. Am $f$ Cardiol 1990;66:926-31.

8 Gurlek A, Dagalp Z, Dervis O, et al. Restenosis after transluminal coronary angioplasty: a risk factor analysis. F Cardiovasc Risk 1995;2:51-5.

9 Benchimol D, Benchimol H, Bonnet J, et al. Risk factors for progression of atherosclerosis six months after balloon angioplasty of coronary stenosis. Am f Cardiol 1990;65:980-5.

10 Myler RK, Topol EJ, Shaw RE, et al. Multiple vessel coronary angioplasty: classification, result and patterns of restenosis in 494 consecutive patients. Cathet Cardiovasc Diagn 1987;13:1-15.

11 Galan KM, Deligonul U, Morton J, et al. Increased frequency of restenosis in patients continuing to smoke cigarettes after percutaneous transluminal in patients continuing to smoke cigarettes after percut

12 Jorgensen B, Endresen K, Forfan K, et al. Attenuated physical exercise capacity in smokers compared with non-smokers after coronary angiocapacity in smokers compared with non-smokers after coronary ang

13 Melkert R, Violaris AG, Serruys PW. Luminal narrowing after percutaneous transluminal coronary angioplasty. A multivariate analysis of clinical, procedural and lesion related factors affecting long-term angiographic outcome in the PARK study. Fournal of Invasive Cardiology 1994;6:160-71.

14 Arora RR, Konrad K, Badhwar K, et al. Restenosis after transluminal coronary angioplasty: a risk factor analysis. Catheterization and Cardiovascular Diagnosis 1990;19:17-22.

\section{STAMPS IN CARDIOLOGY}

\section{Congresses}

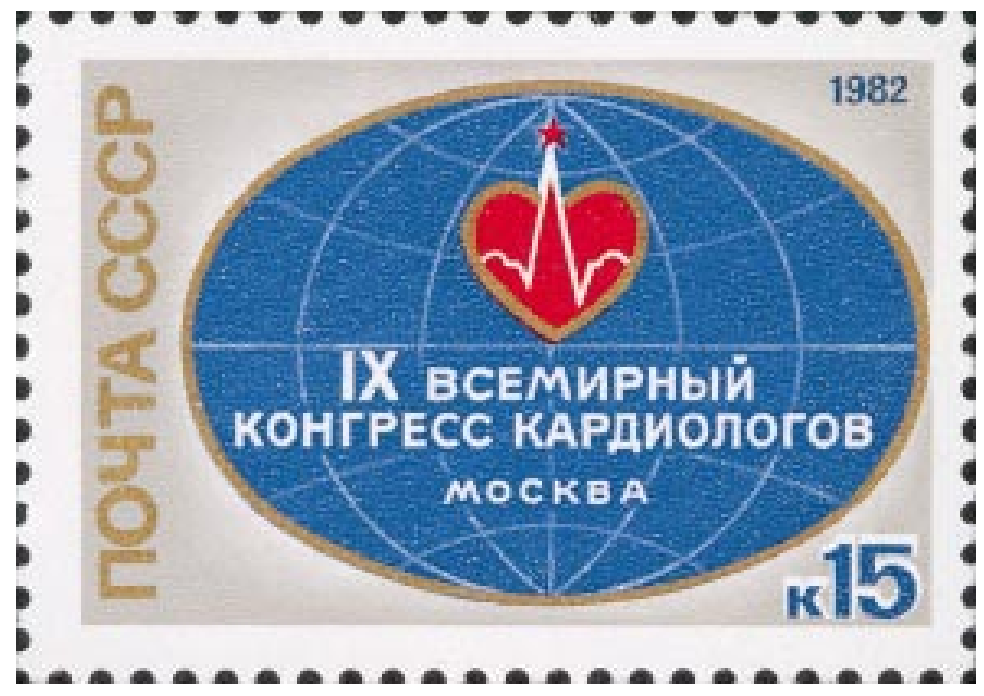

The 9th World Congress of Cardiologists, held in Moscow 20-26th June 1982, was commemorated with the issue of this Russian stamp featuring the heart, globe, and the electrocardiogram. An airmail card was also issued reproducing a design similar to that of the stamp but also showing the portraits of Alexander Maysnikov and Paul Dudley White-the latter as foreign member, Academy of Medical Sciences of the USSR.

M K DAVIES A HOLLMAN 